\title{
A Saline Test Injection Mode Improving the Safety of Using a Power Injector for CT
}

\author{
Shu-Yu Chang ${ }^{1}$, Chia-Hsin Lu ${ }^{1,2}$, I-Chan Chiang ${ }^{2}$, Wei-Shiuan Chung ${ }^{2,3}$ and Tsyh-Jyi Hsieh (iD) ${ }^{2,4,{ }^{*}}$ \\ ${ }^{1}$ Department of Medical Imaging, Kaohsiung Municipal Ta-Tung Hospital, Kaohsiung Medical University, Kaohsiung, Taiwan \\ ${ }^{2}$ Department of Medical Imaging, Kaohsiung Medical University Chung Ho Memorial Hospital, Kaohsiung, Taiwan \\ ${ }^{3}$ Department of Medical Imaging, Kaohsiung Municipal Siaogang Hospital, Kaohsiung Medical University, Kaohsiung, Taiwan \\ ${ }^{4}$ Department of Medical Imaging, Chi Mei Medical Center, Tainan, Taiwan \\ Corresponding author: Department of Medical Imaging, Chi Mei Medical Center, Tainan, Taiwan. Email: tsyhjyi.hsieh@gmail.com
}

Received 2018 October 28; Revised 2019 March 08; Accepted 2019 March 11.

\begin{abstract}
Background: Computed tomography(CT) with rapid injection of contrast medium is important for the diagnosis of many diseases. To obtain good diagnostic accuracy, a power injector is recommended and routinely used to achieve a consistent injection rate of contrast medium. Although rapid contrast injection improves diagnostic accuracy, it increases the risk of extravasation of contrast medium.

Objectives: The aim of this study was to evaluate the extravasation rate of contrast medium using a power injector and determine if using a saline test injection mode can reduce the rate.

Patients and Methods: The records of 10,310 computed tomography (CT) examinations with contrast administration by a dualsyringe power injector were retrospectively reviewed. Before contrast administration, the same volume of saline was injected with a higher injection rate than that for contrast medium and the protocol was defined as "saline test injection mode". The incident reports of patients with extravasations were reviewed and the extravasation rate and prevention rate were calculated.

Results: Extravasations occurred in 12 (0.12\%) of 10310 patients, and follow-up information was available for all patients. Five (41.67\%) of 12 extravasations occurred during the saline test injection period, and the CT examinations were completed after creation of a new venous access. Contrast medium extravasation occurred in only six $(0.06 \%)$ patients. One of the patients with contrast medium extravasation developed compartment syndrome and required decompression surgery. Other cases with extravasation had only mild symptoms and improved within 5 days.

Conclusion: The saline test injection mode may reduce the risk of contrast medium extravasation and improve the safety of using a power injector.
\end{abstract}

Keywords: Multidetector Computed Tomography, Contrast Media, Extravasation of Contrast, Media, Patient Safety, Power Injector

\section{Background}

Computed tomography (CT) is important for the diagnosis of many diseases, and the use of contrast medium is an important part of many examinations. For the evaluation of the hemodynamic changes of lesions and CT angiography, rapid injection of contrast medium is necessary. To obtain good diagnostic accuracy, a power injector is recommended and routinely used to achieve a consistent injection rate of contrast medium $(1,2)$.

Although rapid contrast injection improved diagnostic accuracy, it increases the risk of extravasation of contrast medium (3). The extravasation of contrast medium may lead to severe complications, especially if there is a large amount of leakage, and surgery with or without grafting may be needed $(4,5)$. To reduce the extravasation rate, in addition to training of the staff, testing of the intravenous line with manual injection of normal saline is performed before contrast medium administration $(6,7)$. Even with complete preparation, extravasation rates have been reported to be $0.1 \%-0.9 \%(5,8-13)$. Most cases of contrast extravasation occur in elder patients and those with diabetes mellitus as the veins of these patients may not tolerate the pressure of rapid injection. However, the manual injection of saline for testing cannot sufficiently evaluate the veins because the pressure and injection rate of the power injection cannot be simulated. Injection of saline with power injection is also recommended for testing and may provide adequate evaluation of the veins to determine if contrast injection could be safely performed $(7,14)$. No prior studies, however, have provided the efficiency to ex- 
amine testing vein function using a power injection and saline.

\section{Objectives}

The purposes of this study were to calculate the extravasation rate in using a power injector and to determine if using power injection to inject saline prior to contrast administration could reduce the contrast extravasation rate.

\section{Patients and Methods}

\subsection{Patients}

This study was approved by the institutional review board of our hospital, and because of the retrospective nature, the requirement of informed consent was waived.

The records of all patients who received CT with intravenous injections of contrast medium by a dual syringe power injector between January 1, 2012 and December 31, 2014 were retrospectively reviewed. At our hospital, the standard operating CT procedure is to use a power injector for the intravenous administration of contrast medium, except for those patients in whom suitable venous access cannot be achieved. In this study, patients were excluded if manual administration of contrast medium was performed, the patient was younger than 18 years, and if a peripherally inserted central catheter, central venous line, or port-A-catheter was inserted. Totally, 10,310 CT examinations using a power injector with a saline test mode were enrolled in this study.

\subsection{Acquisition Protocol}

All CT scans were obtained with a 320-row multiple detector CT system (Aquilium One, Toshiba Medical Systems Corporation, Tokyo, Japan) and a dual syringe power injector (Stellant CT injection system, Medrad, Bayer Healthcare, NJ). Nonionic iodinated contrast materials (iohexol 300 [Omnipaque 300; GE Healthcare, Waukesha, Wis]; Iopromide 300 or 370 [Ultravist 300 or 370 ; Bayer Healthcare, $\mathrm{NJ}$ ) were used for all patients.

Before CT examination, all patients who were planned to have a contrast-enhanced CT were examined by one of the well-trained nurses of the CT team to assure adequate peripheral venous access at the antecubital region or forearm. The venous cannulas were checked by administering 5-10 mL of normal saline manually. If the intravenous catheter could not be flushed adequately, or if the patient experienced pain at the injection site, a new peripheral venous access site was created.

\subsection{Saline Test Injection Mode}

The dual syringe power injector provides injection programs to vary rate, volume, and hold that make multiple injections in the same $\mathrm{CT}$ examination possible. In our hospital, the injection protocol is designed with 2 blocks (Figure 1). The first block is for saline test only, and the second block is for contrast administration. The volume of saline used for the test is the same as that of the contrast medium. The injection rate of saline for testing is 0.5 to $1 \mathrm{~mL} / \mathrm{s}$ greater than that of the contrast medium. If extravasation occurs during the saline test, a new venous access is created (Figure 2).

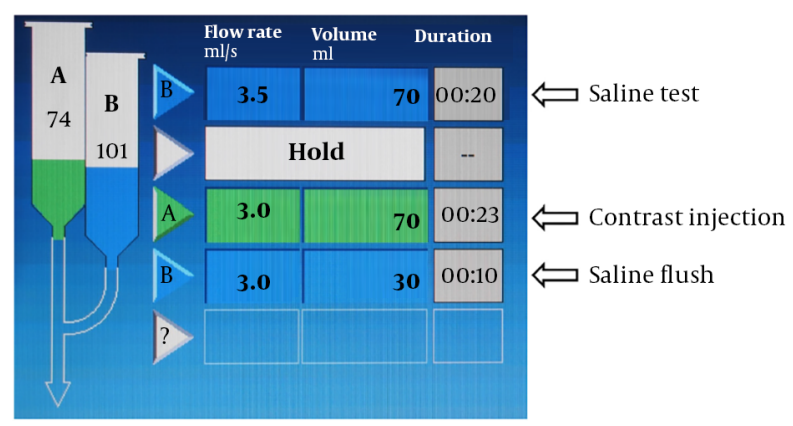

Figure 1. Injection protocol on the screen. This is a sample of CT injection protocol for liver tumor detection.

\subsection{Contrast Administration}

Preheated contrast material (about $37^{\circ} \mathrm{C}$ ) is routinely used, and the rate of contrast medium administration by the power injector is $1-5 \mathrm{~mL} / \mathrm{s}$, based on the types and purposes of the CT examination, e.g., CT angiography, tumor detection and evaluation, and thrombus detection, and the size of the cannulas (20-gauge for $>1.5 \mathrm{~mL} / \mathrm{sec}$ and 22 gauge for no greater than $1.5 \mathrm{~mL} / \mathrm{sec}$ ).

If extravasation was noted, the injection procedure would be interrupted. The clinical criteria for diagnosis of extravasation are as below:

1. Patient complaint pain or swelling at injection site,

2. Abnormal increase or decrease of the injector pressure was noted on the remote monitor of power injector,

3. There was no normal enhancement at the great vessels.

After administration of contrast medium, saline flush with $30 \mathrm{~mL}$ and same flow rate was followed for better enhancement with low iodine doses.

\subsection{Data Collection}

If an extravasation occurred in use of the power injector, immediate symptoms and estimated extravasated volume (EEV) were evaluated. EEV was estimated according to 


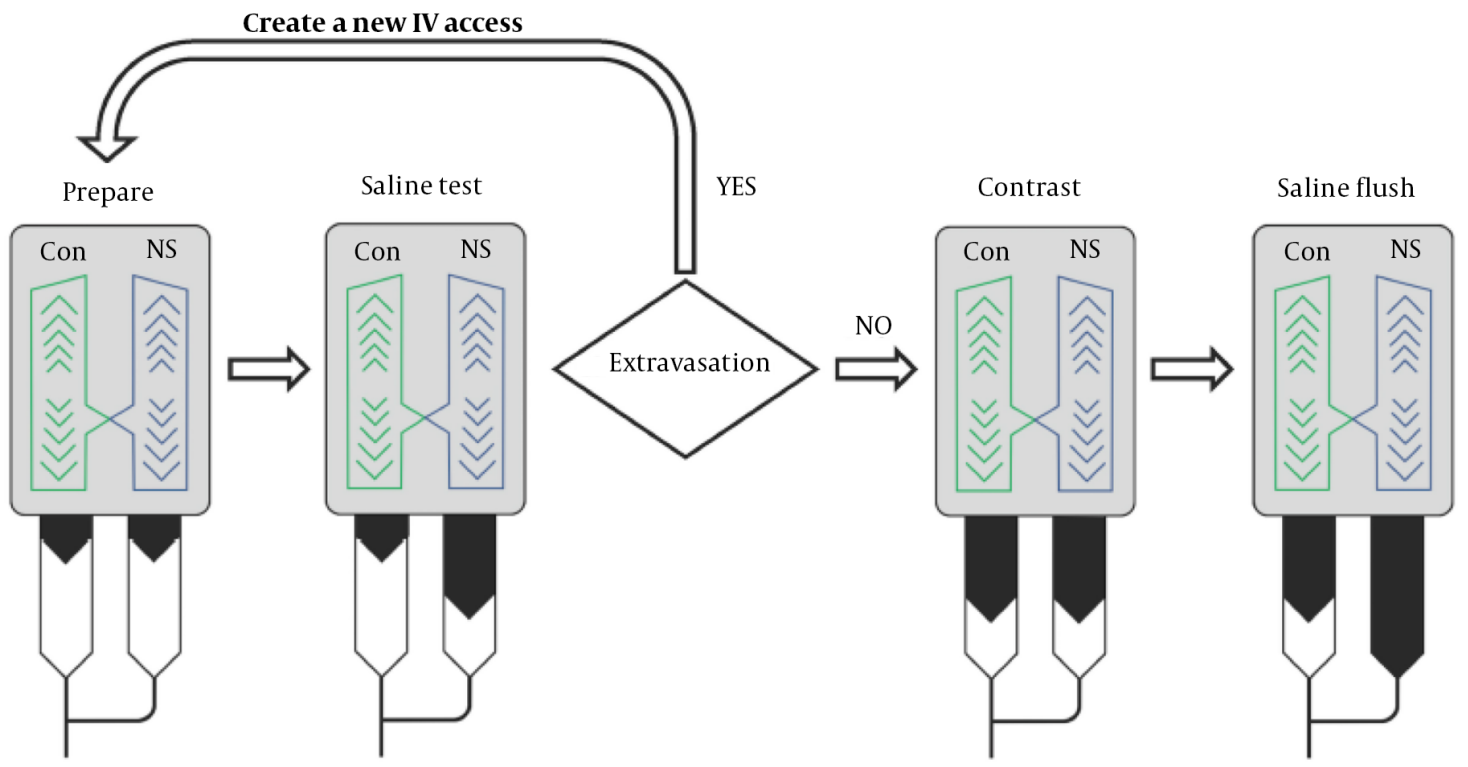

Figure 2. Schematic diagram of the process using power injector

the injection rate and time interval between the episode and stop of injector. The contrast material extravasation incident records were made for each patient with extravasation and these records included patient age and sex, type of extravasated materials (saline test, contrast medium, or saline flush), injection site, intravenous cannula gauge, contrast material injection rate, $\mathrm{EEV}$, immediate symptoms (if any), and treatment. For this study, all the contrast material extravasation incident records were reviewed, and the medical records and the radiology reports of these patients were also reviewed.

\subsection{Statistical Analysis}

Nominal data (sex, injection site, symptoms, treatment) and ordinal data (intravenous cannula gauge) were summarized by counts and percentages. Interval data (age, injection rate) were summarized by means and ranges. The entire study population of unique patients who underwent a CT scan with intravenous contrast was analyzed to calculate the risk (prevalence) of contrast extravasation.

\section{Results}

Between January 1, 2012 and December 31, 2014, 35,613 CT examinations were performed in our hospital, and contrast medium was used in 12,384. Totally, 10,310 CT examinations using a power injector with a saline test mode were enrolled in this study. Twelve extravasations occurred (Table 1) totally, and the overall extravasation rate was $0.12 \%(12 / 10,310)$. One extravasation occurred during the saline flush phase, and no repeat administration was performed. The contrast medium extravasation rate was $0.06 \%$ (6/10310). Five extravasations occurred during the saline test injection phase and the rate in all extravasations was $41.67 \%$.

Of the 12 patients in whom extravasation occurred there were nine females and three males, and in seven cases (58.33\%), the intravenous cannula was placed in the radiology department and in five cases (41.67\%) in the ward. Half of the extravasation cases had the intravenous cannula placed in the antecubital veins and the other half in the forearm veins. In the cases with extravasation, the size of the cannula in seven cases (58.33\%) was 20-gauge, and in the other cases was 22-gauge. The average injection rate in the cases with extravasation was $2.25 \pm 0.92 \mathrm{~mL} / \mathrm{s}$ (range, 1.2 - $4.5 \mathrm{~mL} / \mathrm{s}$ ). The average EEV was $39.58 \pm 23.01 \mathrm{~mL}$ (range, $20-70 \mathrm{~mL}$ ). Local tenderness or swelling was noted in all cases. Local pain was noted in six cases only. One of the patients with extravasation developed compartment syndrome and received a decompression surgery. There were no complications in the other patients with extravasation.

\section{Discussion}

The results of the current study indicate that the saline test injection method can reduce the contrast extravasation rate using an automated intravenous contrast 


\begin{tabular}{|c|c|c|c|c|c|c|c|c|c|}
\hline \multirow{2}{*}{$\begin{array}{l}\text { Patient } \\
\text { No./Age } \\
\text { (y)/Sex }\end{array}$} & \multirow{2}{*}{$\begin{array}{l}\text { Cannula size } \\
\text { (G)/location }\end{array}$} & \multirow{2}{*}{$\begin{array}{l}\text { Injection } \\
\text { rate, } \\
\mathrm{mL} / \mathrm{sec}\end{array}$} & \multirow{2}{*}{$\begin{array}{l}\text { Staff sited the } \\
\text { cannula }\end{array}$} & \multicolumn{3}{|c|}{ Type of extravasation and $\mathrm{EEV}^{*}, \mathrm{~mL}$} & \multirow[t]{2}{*}{ Symptoms } & \multirow[t]{2}{*}{ Treatment } & \multirow{2}{*}{$\begin{array}{l}\text { Sequelae/follow- } \\
\text { up time, } \\
\text { day }\end{array}$} \\
\hline & & & & $\begin{array}{l}\mathrm{Ns}^{\#} \\
\text { test }\end{array}$ & Contrast injection & $\begin{array}{c}\text { NS } \\
\text { flush }\end{array}$ & & & \\
\hline $1 / 67 / \mathrm{F}$ & 22/Forearm & 1.2 & Ward & 20 & - & - & Local swelling and pain & Conservative & $\mathrm{Ni} / 5$ \\
\hline $2 / 50 / F$ & 20/Forearm & 3 & Radiology & 70 & - & - & Local tenderness and numbness & Conservative & $\mathrm{Nil} / 4$ \\
\hline 3/75/M & 22/Antecubital & 2.3 & Radiology & 20 & - & - & Local tenderness and numbness & Conservative & $\mathrm{Nil} / 2$ \\
\hline $4 / 70 / F$ & 20/Antecubital & 4.5 & Radiology & - & 55 (iopromide 370) & - & Local tenderness and numbness & Conservative & $\mathrm{Nil} / 3$ \\
\hline $5 / 44 / M$ & 22/Antecubital & 1.5 & Radiology & - & 70 (iopromide 300) & - & Local swelling and pain & Conservative & $\mathrm{Nil} / 3$ \\
\hline 6/75/F & 22/Forearm & 2 & Radiology & 20 & - & - & Local swelling & Conservative & $\mathrm{Nil} / 2$ \\
\hline $7 / \mathbf{4 2} / \mathbf{F}$ & 22/Forearm & 2.3 & Radiology & 60 & - & - & Local swelling & Conservative & $\mathrm{Nil} / 1$ \\
\hline $8 / 73 / \mathbf{F}$ & 20/Antecubital & 2.5 & Ward & . & - & 10 & Local swelling and pain & Conservative & $\mathrm{Nil} / 1$ \\
\hline 9/57/M & 20/Antecubital & 1.5 & Ward & . & 30 (iopromide 300) & . & Local swelling and pain & Conservative & $\mathrm{Nil} / 1$ \\
\hline $10 / 60 / F$ & 20/Antecubital & 2.5 & Radiology & . & 20 (iopromide 300 ) & . & Local tenderness & Conservative & $\mathrm{Nil} / 4$ \\
\hline $11 / 56 / \mathrm{F}$ & 20/Forearm & 2.5 & Ward & - & 70 (iopromide 300) & - & Local swelling and pain, cold skin & Decompression & $\mathrm{Nil} / 30$ \\
\hline $12 / 71 / \mathrm{F}$ & 20/Forearm & 1.2 & Ward & - & 20 (iopromide 300 ) & . & Local swelling and pain & Conservative & $\mathrm{Nil} / 1$ \\
\hline
\end{tabular}

Abbrevitions: ENV estimated extasated volume; F, female; M, male Nil, nothing: NS, normal saline

medium power injector. The overall extravasation rate in our study was $0.12 \%$, and the rate of extravasation with contrast medium was only $0.06 \%$, which is lower than previously reported extravasation rates (5, 8-13). The difference between the overall extravasation rate and the contrast extravasation rate means that the saline test injection method can detect patients with a higher risk of contrast medium extravasation and reduce the contrast medium extravasation rate by $41.67 \%$. To our knowledge, there are no published data that demonstrate that the contrast medium extravasation risk can be reduced by using a saline test injection.

The use of a power injector provides greater information for the CT examination $(1,15,16)$. However, a faster injection rate may lead to contrast extravasation and potentially severe complications $(3,5,10,17)$. Prior studies reported that the incidence of intravenous contrast extravasation using a power injector range from $0.1 \%$ to $0.9 \%$ (5, $8-13)$. In our study, the overall extravasation rate was $0.12 \%$, which is at the low end of that reported in prior research. The low incidence in our study may be due to the relatively low injection rate of contrast medium via the power injector $(1-5 \mathrm{~mL} / \mathrm{s})$. Phantom studies have shown that the contrast medium injection pressure increased with an increase in the injection rate (18). In our hospital, the injection rate may be reduced if there is deemed to be poor venous tolerance at the injection site, which is routinely checked by our stuff. A lower injection rate may result in a lower injection pressure, which may decrease the incidence of extravasation in patients with potentially poor venous tolerance.

Although the currently available iodine-based contrast media generally are considered to be safe, extravasation of the contrast medium may lead to complications, especially when using a power injector. Most patients with contrast extravasation are asymptomatic, or have mild symptoms such as swelling, tightness, stinging, burning pain, edema, erythema, or tenderness at the injection site. For these patients, conservative treatments with elevation of the affected extremity and a warm or cold compress are recommended. Only rarely do serious complications such as compartment syndrome, skin ulceration, and tissue necrosis, which may require surgical intervention, occur. In our study, only one patient developed compartment syndrome after contrast extravasation and received surgery treatment. No long-term sequelae were noted in this patient. Other patients in whom extravasation occurred had only mild symptoms and no surgical intervention was needed. All of the patients with normal saline extravasation, including the saline test phase and saline flush phase, experienced only mild symptoms as there was no contrast medium present.

Prevention of contrast extravasation at the intravenous access site important for the safe use of contrast media. Previous studies have examined potential risk factors for contrast medium extravasation including sex, age, injection rate, cannula size, cannula location, and the type of staff who inserted the cannula $(6,11,19)$. At our hospital, it is standard procedure to use a cannula of $22 \mathrm{G}$ or greater, and only place it in an antecubital or forearm vein. The results of our study showed that the incidence of extravasation did not vary with cannula size or cannula location. Similar to a previous study, the incidence of extravasation did not increase with an increase in the injection rate in our study (11). In addition, the incidence of extravasation was similar regardless of the person inserting the cannula 
(19).

For decreasing the extravasation rate, the American College of Radiology (ACR) and the Society for Pediatric Radiology (SPR) practice guidelines for the use of intravascular contrast media suggests that the intravenous access should be examined to verify that the catheter is appropriate for the injection (7). Methods for determining suitability include checking for the backflow of blood, saline flush, and/or test injection with a power injector. In most of the previous studies regarding contrast administration, test injections were performed by manual push only. Only one study used a saline test injection by a power injector to estimate the contrast medium pressure in pediatric CT and suggest that a saline test injection may be useful (20).

Different from the previous study, we performed the saline test by manual pushing and a power injector. In our study, five (41.67\%) of all extravasations occurred with the saline test injection, and no severe complications were noted in these patients. Compared to iodinated contrast medium, normal saline has less toxicity and is suitable for testing (21). However, a manual push saline test does not reach the pressure of using a power injector, especially the higher pressure at a higher injection rate. For simulating the conditions of contrast medium injection, we use the same volume of normal saline as would be used with the contrast medium, but with a higher injection rate. We used a higher injection rate with the saline test because normal saline develops less pressure than iodinated contrast medium at the same injection rate. Even a saline injection rate that is $0.5-1 \mathrm{~mL} / \mathrm{s}$ greater than that of the contrast medium injection may not attain the same pressure.

Several limitations of this study should be noted. First, this was a single-hospital study, which might limit the generalizability of the results. Second, the study data were collected retrospectively. Although guidelines for highquality retrospective data collection were followed, the details of all participants could not be evaluated for risk factor detection. Third, subtle or minimal extravasation without significant symptoms may not be recorded. Although the post-injection condition of the patients was evaluated by our well-trained nurses, only patients with focal discomfort, focal mass or poor enhancement in the post-enhanced images would be rechecked and recorded. Forth, we did not perform a power statistic calculation because we did not have a control group. In addition, we did not perform an in vitro study to determine if the amount of normal saline used for the power injector test was adequate.

In conclusion, a saline test injection using a power injector is simple to perform and may reduce the risk of contrast medium extravasation.

\section{Footnotes}

Authors' Contributions: Study concept and design: ShuYu Chang, Chia-Hsin Lu, I-Chan Chiang, Wei-Shiuan Chung, and Tsyh-Jyi Hsieh; acquisition of data: Shu-Yu Chang, ChiaHsin Lu, and Tsyh-Jyi Hsieh; analysis and interpretation of data: Shu-Yu Chang, I-Chan Chiang, and Tsyh-Jyi Hsieh; drafting of the manuscript: Shu-Yu Chang, Chia-Hsin Lu, I-Chan Chiang, Wei-Shiuan Chung, Tsyh-Jyi Hsieh; critical revision of the manuscript for important intellectual content: Shu-Yu Chang and Tsyh-Jyi Hsieh; statistical analysis: Shu-Yu Chang, Wei-Shiuan Chung, and Tsyh-Jyi Hsieh; administrative, technical, and material support: Shu-Yu Chang, Chia-Hsin Lu, and Tsyh-Jyi Hsieh; study supervision: Chia-Hsin Lu and Tsyh-Jyi Hsieh

Conflict of Interests: There is no conflict of interest or industry support of the project.

Ethical Considerations: This study was approved by the Institutional Review Board of Kaohsiung Municipal Ta-Tung Hospital, Kaohsiung Medical University, and because of the retrospective nature the requirement of informed consent was waived. The IRB Number is "KMUHIRBE-20150064". The certificate is attached as one of Figures.

Financial Disclosure: None declared.

Funding/Support: This research did not receive any specific grant from funding agencies in the public, commercial, or not-for-profit sectors.

\section{References}

1. Schoellnast H, Tillich M, Deutschmann HA, Stessel U, Deutschmann MI, Schaffler GJ, et al. Improvement of parenchymal and vascular enhancement using saline flush and power injection for multiple-detector-row abdominal CT. Eur Radiol. 2004;14(4):659-64. doi: 10.1007/s00330-003-2085-3. [PubMed: 14566425].

2. Ishida M, Schuster A, Morton G, Chiribiri A, Hussain S, Paul M, et al. Development of a universal dual-bolus injection scheme for the quantitative assessment of myocardial perfusion cardiovascular magnetic resonance. J Cardiovasc Magn Reson. 2011;13:28. doi: 10.1186/1532-429X13-28. [PubMed: 21609423]. [PubMed Central: PMC3118114].

3. Jacobs JE, Birnbaum BA, Langlotz CP. Contrast media reactions and extravasation: Relationship to intravenous injection rates. Radiology.1998;209(2):411-6. doi: 10.1148/radiology.209.2.9807567. [PubMed: 9807567].

4. Bellin MF, Jakobsen JA, Tomassin I, Thomsen HS, Morcos SK, Thomsen HS, et al. Contrast medium extravasation injury: Guidelines for prevention and management. Eur Radiol. 2002;12(11):2807-12. doi: 10.1007/s00330-002-1630-9. [PubMed: 12386778].

5. Wang CL, Cohan RH, Ellis JH, Adusumilli S, Dunnick NR. Frequency, management, and outcome of extravasation of nonionic iodinated contrast medium in 69,657 intravenous injections. Radiology. 2007;243(1):80-7. doi: 10.1148/radiol.2431060554. [PubMed:17392249].

6. Beckett KR, Moriarity AK, Langer JM. Safe use of contrast media: What the radiologist needs to know. Radiographics. 2015;35(6):1738-50. doi: 10.1148/rg.2015150033. [PubMed: 26466182].

7. American College of Radiology. Acr-spr practice parameter for the use of intravascular contrast media. Radiology ACo ed. revised (resolution 5). 2017 
8. Shaqdan K, Aran S, Thrall J, Abujudeh H. Incidence of contrast medium extravasation for CT and MRI in a large academic medical centre: A report on 502,391 injections. Clin Radiol.2014;69(12):1264-72. doi:10.1016/j.crad.2014.08.004. [PubMed: 25248291].

9. Cohan RH, Ellis JH, Garner WL. Extravasation of radiographic contrast material: Recognition, prevention, and treatment. Radiology. 1996;200(3):593-604. doi: 10.1148/radiology.200.3.8756899. [PubMed: 8756899].

10. Federle MP, Chang PJ, Confer S, Ozgun B. Frequency and effects of extravasation of ionic and nonionic CT contrast media during rapid bolus injection. Radiology. 1998;206(3):637-40. doi: 10.1148/radiology.206.3.9494479. [PubMed: 9494479].

11. Wienbeck S, Fischbach R, Kloska SP, Seidensticker P, Osada N, Heindel W, et al. Prospective study of access site complications of automated contrast injection with peripheral venous access in MDCT. AJR Am J Roentgenol.2010;195(4):825-9. doi: 10.2214/AJR.09.3739. [PubMed: 20858804].

12. Moreno CC, Pinho D, Nelson RC, Sahani DV, Jenkins M, Zabrycki $\mathrm{M}$, et al. Lessons learned from 118,970 multidetector computed tomographic intravenous contrast material administrations: Impact of catheter dwell time and gauge, catheter location, rate of contrast material administration, and patient age and sex on volume of extravasate. J Comput Assist Tomogr. 2013;37(2):286-8. doi: 10.1097/RCT.ob013e31828211da. [PubMed: 23493221].

13. Dykes TM, Bhargavan-Chatfield M, Dyer RB. Intravenous contrast extravasation during CT: A national data registry and practice quality improvement initiative. I Am Coll Radiol. 2015;12(2):183-91. doi: 10.1016/j.jacr.2014.07.021. [PubMed: 25245788].

14. Hardie AD, Kereshi B. Incidence of intravenous contrast extravasation: Increased risk for patients with deep brachial catheter placement from the emergency department.Emerg Radiol.2014;21(3):235-8. doi: 10.1007/s10140-013-1185-x. [PubMed: 24395398].
15. Shuman WP, Adam JL, Schoenecker SA, Tazioli PR, Moss AA. Use of a power injector during dynamic computed tomography. J Comput Assist Tomogr. 1986;10(6):1000-2. doi: 10.1097/00004728-19861100000020. [PubMed: 3782538].

16. Pinto A, Niola R, Tortora G, Ponticiello G, Russo G, Di Nuzzo L, et al Role of multidetector-row CT in assessing the source of arterial haemorrhage in patients with pelvic vascular trauma. Comparison with angiography. Radiol Med. 2010;115(4):648-67. doi: 10.1007/s11547-0100494-0. [PubMed: 20082229].

17. Nelson RC, Anderson FA Jr, Birnbaum BA, Chezmar JL, Glick SN. Contrast media extravasation during dynamic CT: Detection with an extravasation detection accessory. Radiology. 1998;209(3):837-43. doi: 10.1148/radiology.209.3.9844684. [PubMed: 9844684].

18. Ruess L, Bulas DI, Rivera O, Markle BM. In-line pressures generated in small-bore central venous catheters during power injection of CT contrast media. Radiology. 1997;203(3):625-9. doi: 10.1148/radiology.203.3.9169679. [PubMed: 9169679].

19. Kingston RJ, Young N, Sindhusake DP, Truong M. Study of patients with intravenous contrast extravasation on $\mathrm{CT}$ studies, with radiology staff and ward staff cannulations. J Med Imaging Radiat Oncol. 2012;56(2):163-7. doi: 10.1111/j.1754-9485.2012.02355.x. [PubMed: 22498188].

20. Abe S, Mizuno N, Tani S, Nishikawa M, Yabunaka K, Mizuta M, et al. Effectiveness of the new injection program 'saline test injection mode' for use power injector in pediatric contrast CT. Australas Phys Eng Sci Med. 2013;36(3):347-54. doi: 10.1007/s13246-013-0207-z. [PubMed: 23797765].

21. Cohan RH, Dunnick NR, Leder RA, Baker ME. Extravasation of nonionic radiologic contrast media: Efficacy of conservative treatment. Radiology. 1990;176(1):65-7. doi: 10.1148/radiology.176.1.2353113. [PubMed: 2353113]. 